\title{
Recognition memory for elements of sentences
}

\author{
ALLAN G. REYNOLDS and PAUL W. FLAGG \\ Dartmouth College, Hanover, New Hampshire 09755
}

\begin{abstract}
Although several theoretical positions and a variety of empirical tasks indicate the importance of verbs to sentences, nouns are generally recalled and recognized better in memorial tasks. Three main models can be identified to explain this discrepancy ("Fillenbaum's paradox"). To try to resolve this paradox, several experiments explored the efficiency of various sentence elements as cues in recognition memory. In Experiment I, concreteness of the stimuli did not interact with the type of distractor; however, verb phrase changes were harder to recognize than noun phrase changes when synonym distractors were used. This result was replicated in a forced-choice recognition paradigm (Experiment II) and with whole sentences where the derivational similarity of verbs and nouns was controlled (Experiment IV). The effect could not be attributed to characteristics of the English language (Experiment III) or to superior memory for form information in nouns (Experiment V). The total results are interpreted as suggesting that subjects process different parts of a sentence to different semantic levels, with verbs receiving more semantic representation and nouns more orthographic or phonological representation. The results are taken as support for a "semantic encoding model" of Fillenbaum's paradox.
\end{abstract}

On intuitive grounds, it is generally felt that the verb carries a heavy burden for conveying meaning in a sentence. This common-sense observation is paralleled by several recent theoretical linguistic approaches emphasizing the importance of verbs. Chafe (1970), for example, has offered an entire utterance model based on the central role of verbs; in Chafe's approach, the verb is the semantic core of the sentence and other forms of speech derive their meaning from their relations to that verb.

Several psychological models of sentence processing also emphasize the role of verbs. As Wearing (1974) pointed out, the verb should be very important for surface structure models (Martin \& Roberts, 1966; Yngve, 1960) of processing and memory because the placement of verbs directly affects the mean depth of a sentence; this variable has been shown to be important in a variety of tasks (Martin \& Roberts, 1966; Wearing, 1970, 1972; Wearing \& Crowder, 1971). Generative linguistic models (Fodor, Garrett, \& Bever, 1968 , p. 459) also view the verb as at least as important to deep structure as other parts of speech, and it is well known that deep structural relations seem to be critical in certain memorial tasks (Blumenthal, 1967;

The research reported in this article was sponsored in part by Grant OEG-1-71-0101 from the U.S. Office of Education, Department of Health, Education, and Welfare. The opinions expressed herein, however, do not necessarily reflect the position or policy of the U.S. Office of Education, and no official endorsement by the U.S. Office of Education should be inferred. A version of this paper was presented at the annual meetings of the American Psychological Association, Montreal, August 1973. We wish to thank Wayne Henshaw for collecting the data for Experiments II and III, Tom Tighe for suggesting Experiment V, and George Potts for his helpful comments on an earlier draft of this paper. Reprint requests should be sent to Allan G. Reynolds, Department of Psychology, Dartmouth College, Hanover, New Hampshire 03755.
Jarvella, 1971; Sachs, 1967; Weisberg, 1969; Townsend \& Saltz, Note 1).

A variety of researchers have shown empirically how manipulations of verbs can influence sentence processing. The effects of varying verb transitivity have been demonstrated by Bacharach, Kellas, and McFarland (1972), Polzella and Rohrman (1970), and Rohrman (1970); for complete sentence recall Thios (1975) has shown that, when verbatim responses are used in a cuedrecall task, sentences with specific verbs are recalled better than sentences with general verbs. Using a paired-associate task with nouns as both stimuli and responses, Bower (1970) has reported similar findings when subjects were instructed to image a scene of two objects interacting in some way or to image the two objects as independent entities in their imagined space (for example, as pictures hanging on opposite walls of a room). In a cued recognition test, the interactive imagery subjects recalled $71 \%$, whereas the subjects in the other condition recalled only $46 \%$ of the response terms. Thus, the introduction of an action (i.e., verb) component enhanced performance markedly. This may be because the verbs act as the center point around which the other items can pivot.

The most impressive evidence for the primacy of verbs comes from a variety of rating and sorting tasks. The importance of verbs, as compared to other sentence elements, in judging connotative meaning of sentences was reported by Gollub (1968) and Heise $(1969,1970)$. Healy and Miller (1970) have also shown the effects of verbs on judgments of denotative meaning; in their study, verb similarity was the single variable most predictive of overall sentence similarity. In the same vein, Healy and Miller (1971) found that removing a verb from a sentence has graver effects on the sentence's grammatical acceptability than the removal of a noun. 
Similar evidence on the importance of the verb with regard to sentence meaning comes from a study by Gladney and Krulee (1967). These authors were interested in the perception of errors in simple sentences. To produce errors in a sentence, they replaced the subject, object, or verb with an adjective or an adverb. The subjects' viewing time in perceiving these altered sentences indicated that changes in the verb were more disruptive than a change in either noun, regardless of whether the substitution was an adjective or an adverb. Horowitz and Prytulak (1969) argued, since this finding is counter to their position which stresses the importance of nouns, that one cannot make a clear interpretation of this effect, since the verb changes resulted in a greater error rate than the noun changes. To use their example: "The foreigner lonesome the speech of the visitor" (verb change) is clearly more anomalous than "The drunken timidly told them a ridiculous anecdote" (noun change). However, this is just the effect one would expect if the verb is indeed more central to the meaning of a sentence than are nouns.

Healy and Miller (1970) proposed a theatrical metaphor to describe the role of verbs and nouns in sentences: "The main verb of a sentence defines the plot; the subject merely indicates one of the actors" (p. 371). However, it appears that, when subjects know that they must remember something about the play (sentence), it is the actors (nouns) that are remembered, rather than the plot (verb). Contrasting sharply with the above theoretical and empirical support for the primacy of verbs is a wealth of evidence derived from memorial tasks indicating the striking inferiority of verbs in recognition, recall, and cueing tasks. For example, Wearing (1970) found verb recall to be inferior to agent and object recall after both $30-\mathrm{sec}$ and 48 -h delays; verbs were also less powerful recall cues. Horowitz and Prytulak (1969) and James (1972) have presented similar results. Using recall tasks, Martin, Roberts, and Collins (1968) and Martin and Walter (1969) have also found generally poorer recall of verbs. Kintsch (1974, Chapter 7, Experiments 1 and 2) extended and qualified the general findings that verbs are recalled poorer than subjects or objects. For all simple subject-verb-object sentences used, as well as more complex active sentences involving indirect objects, the expected findings obtained. However, for two of the five sentence types which involved multiple semantic propositions, recall of the verb was intermediate between that of the subject and object. As well, one sentence type (a subject-verbobject-indirect object sentence in the passive) gave superior recall for the verb. These results must be interpreted as a warning about the total generality of the subject-object-verb recall order reported in earlier studies.

Wearing (1973) found that verbs were the poorest recalled, of all parts of speech, for sentences of 5,7 ,
9,11 , and 13 words; this inferiority was most marked for sentences longer than seven words. Wearing suggested that when the span of memory is exceeded, subjects recode the verb into a more abstract form (perhaps as a set of markers or components relating the subject and object), while subjects store the subject and object more directly (perhaps as images). Reid (1974) likewise argued that the verb has no direct representation in memory. This view is in sharp contrast to Chafe (1970), who argued that noun meaning is determined by verb characteristics.

Thios (1975) offered an explanation similar to Wearing's to explain his finding that verbs were poorer recall cues than subjects; he noted that when verb synonyms were allowed as correct responses, these synonyms were much more common for general ("made") rather than specific ("carved") verbs. Thios suggested that fewer noun attributes were specified by general verbs, therefore less information was extracted. As a result, subjects were more likely to generate synonyms at recall time. Gomulicki (1956), dealing with connected prose passages, also found verbs to be recalled poorer than nouns; this pattern was reversed when synonyms were allowed as correct answers.

A theoretical explanation similar to Wearing's has been advanced by Begg and Paivio (1969); Begg and Paivio have argued, at least with respect to concrete material, that subjects form a mental picture (i.e., image) of the scene depicted in the sentence. This implies a natural ordering for form classes, with respect to recall (and probably recognition): "Recall of sentence nouns merely requires labeling the pictorial objects, recalling adjectives requires that the subject describe the objects, recalling verbs requires labeling of an inferred action in which the objects are engaged, and recalling adverbs requires description of that action. Thus, there should be more semantic distortion in recall of sentences in order from nouns to adverbs" (Begg \& Paivio, 1969, p. 826). The prediction for abstract material is less clear, but such an ordering may not exist, as the sentence components seem to be more closely tied to the surface structure ordering.

However, another argument could be offered to explain why some cues are more effective than others. This argument is based on the importance of an item to the semantic structure of a sentence. Items that are of the most importance are processed to a "deeper" or more "meaningful" level than less important items. The nature of this semantic structure is unspecified at present, but Kintsch (1972), Rumelhart, Lindsay, and Norman (1972), and others have made some suggestions about the nature of this semantic memory. The idea that certain elements receive more processing than others is in agreement with the position of Gomulicki (1956), who argued that various parts of a passage are actively, and perhaps unwittingly, selected by subjects for differential analysis by a process he called mnemic 
abstraction. This idea of different levels of processing is similar to that recently presented by Craik and Lockhart (1972).

Fillenbaum (1970) has tried to explain the apparent paradoxical findings about the role of verbs in sentences by suggesting that "It is quite possible that the way in which a sentence is analyzed and stored in a memorial task . . . may be quite different in important respects from the way in which it is processed in a task which requires full understanding of the sentence." In support of this contention, Anderson and Hidde (1971), in an incidental learning task where a subject was the cue for verb and object recall, found no superiority in recall of object nouns. Wearing (1974), dubbing this problem "Fillenbaum's paradox," noted that memory tasks should tap underlying meaning and similarity judgment tasks should do the same. Wearing suggested that the source of the paradox might lie in radically different sentential materials being used in the two types of tasks. To circumvent this possible confound, Wearing used a common set of sentences in both a semantic sorting task and a free recall memory task; the words (subject, verb, object) varied on Osgoodian potency. Wearing's sorting results replicated Healy and Miller (1970); $77 \%$ of the subjects sorted principally on the basis of the verb; there were no differences due to potency of the words. In the recall task, for lowpotency sentences, there was no difference for recallability of subjects, verbs, and objects; for high-potency sentences, verbs were poorest recalled. Wearing suggested that the functional significance of the verb in sentences changes in the course of sentence processing, since the memory task required the reconstruction of the sentence minutes after presentation, while the judgment task imposed no memory load. This explanation, of course, is similar to that advanced earlier by Wearing (1973) and Fillenbaum (1970). Wearing further proposed that if the value of the verb, in its surface structure form, does change over the time course of the memory experiment, it might be wise to examine immediate memory, i.e., at the point of comprehension. Here, verb-noun differences should be erased, or even reversed. In fact, Wearing (1971), using a divided at tention technique, found verb recall to be superior to noun recall.

Three main theoretical explanations for the inferiority of verbs in memorial tasks may be abstracted from the above speculations. One we may call the "verb decomposition model;" this is exemplified by Wearing's suggestion that the verb changes form over the course of the memory task. Begg and Paivio's imagery model may be viewed as a paraphrase of Wearing's approach. Second, the Gomulicki and Craik and Lockhart approaches could be called the "levels of processing model" or, alternatively, the "semantic encoding model;" this is similar to the first model, with the exception that the changes in storage of the verb occur not over time but at the point of compre- hension. Finally, Fillenbaum's "task demand model" states that the mere fact of intentional learning in a memory task reduces the necessity of semantic analysis inherent in a sorting or judgment task.

Nouns and verbs seem clearly to have special importance in phrase and sentence memory. However, other elements may be important candidates as efficient cues for recognition as well. In addition, most of the studies underlying these three models have used simple S-V-O sentences in the active voice as test materials. Therefore, the present study will examine how the relationship between nouns and verbs, and their modifiers in phrases, may be affected in recognition by several factors such as the voice of the sentence and the sentence's concreteness. Hopefully, these data will help resolve Fillenbaum's paradox and allow a choice among these three similar explanations of the verb's role in sentence memory. Also, by replacing various words in the recognition phrase with either synonyms or unrelated words, we seek to gain a better understanding of how the different components of a sentence are stored in memory.

\section{EXPERIMENT I}

\section{Method}

Subjects. A total of 80 subjects participated in the study; 32 subjects served in the experiment proper, while 48 subjects helped generate stimulus sentences. All subjects were students in introductory psychology at Dartmouth College and served for course credit.

Design. The experiment used a recognition memory paradigm. Five sentences were presented in a block for the subject to study; after an interpolated activity (reciting the alphabet backward), a phrase (either unchanged or altered) from one of the five sentences was presented; the subject's task was to signify if he had seen this target (test) phrase in the sample of five sentences.

The experiment involved a factorial design with two betweensubjects factors and three repeated-measures factors. The two between-subjects factors were Concreteness (concrete or abstract sentences) and Voice (sentences were either active or passive). Eight subjects were tested in each of the four cells formed by the two factors. There were three repeated-measures factors: The Cue Phrase Presented could be either the subject phrase, verb phrase, or object phrase; the Part of Speech Changed could be either the modifier or head (noun or verb) in the phrase; the Type of Change could be a change to a synonym or to an unrelated word.

Materials. All sentences had the general form article, adjective, noun, adverb, verb, article, adjective, noun (e.g., The deep anger quickly caused the noisy outburst). All sentences werc reversible, that is, the subject and object phrases could be interchanged without creating a nonsense sentence. The experimenters and an assistant each examined a large pool of possible test sentences generated by subjects, and discarded any that were anomalous. Of the remaining sentences, those which all three people agreed contained nouns and verbs with physical referents were classified as concrete. Those with no physical referents were classified as abstract. After the concrete and abstract sentences of the above form had been chosen, every other sentence had the subject and object phrases interchanged to avoid any possible bias in sentence construction. Each sentence was then transformed into the passive form and checked for meaningfulness. From this pool, 48 concrete and 48 abstract sentences were chosen. Altered versions of 24 of the 48 test sentences for each kind (concrete and abstract) were constructed 
in the following manner. Eight alternates had a change performed in the subject phrase, eight in the verb phrase, and eight in the object phrase (Cue Phrase Presented factor). Within each group of eight alternate sentences, four had the modifier (adjective or adverb) changed and four had the head (noun or verb) changed (Part of Speech Changed factor). Within each of these four sentences, two of the changes involved replacement by synonyms; the other two changes involved extraneous (neither synonymous nor antonymous) words. All replacement words were matched to the original words as closely as possible on Thorndike-Lorge frequency. Filler sentences for each presentation block had the same grammatical form and number of words as the 24 critical sentences. No filler sentence was used more than once. These filler sentences were selected such that they shared no words other than articles with each other or with the target sentences.

Procedure. Testing of subjects took place at a computer terminal connected to the Dartmouth time-sharing system; after a subject was seated at the terminal and had read the instructions, a block of five sentences was presented. One of these five was the target sentence; four were fillers. A block was exposed for $20 \mathrm{sec}$, after which the subject was presented with a randomly selected letter of the alphabet. The subject was instructed to say the alphabet backward from this start point. After $5 \mathrm{sec}$ of alphabet pronunciation, the subject was presented with a cue phrase which may or may not have appeared in one of the sentences in the preceding block of five. Half the time the cue phrase was identical to a phrase presented in one of the preceding five sentences; half the time the phrase had been altered in some manner. For each subject, different patterns of old and changed phrases were generated; that is, the specific sentences used as sources for test phrases were counterbalanced across subjects. After the cue phrase was presented, the subject was asked to decide whether he had seen this phrase in the preceding five sentences (old/new judgment).

\section{Results and Discussion}

OLD cue phrases. The $d^{\prime}$ measure of signal detectability was used to correct for guessing or response bias. Each subject's proportion correct (when the cue phrase was OLD) and proportion incorrect (when the cue phrase was NEW) for all NEW phrases were converted to average $d^{\prime}$ scores. Each subject obtained these $d^{\prime}$ scores, one for each of the three levels of the repeated-measures factor in this analysis (Cue Phrase Presented). The other two factors included in the analysis were Concreteness and Voice of the sentences. There were two significant effects in this analysis. Concrete cue phrases were more easily detectable as OLD (.70) than abstract cue phrases $(.59, \mathrm{~F}=26.77$, $\mathrm{df}=1 / 28, \mathrm{p}<.001, \mathrm{MSe}=.62)$. As well, the two noun phrases (subject phrase and object phrase) were more often detected correctly as OLD than the verb phrase $(\mathrm{F}=5.99, \mathrm{df}=2 / 56, \mathrm{p}<.005, \mathrm{MSe}=.66)$. In analysis of simple proportions correct, uncorrected for guessing, both of the above effects narrowly missed standard significance levels $(p=.077$ and $p=.064)$. For the Cue Phrase Presented effect, the mean proportions correct were .64 (subject), .58 (verb), and .68 (object). The failure to obtain a significant effect for Voice is mildly surprising, but is in agreement with the results of Wearing (1970).

NEW cue phrases. The proportions correct for judging NEW phrases were analyzed in a five-way analysis of variance, where the factors were Voice, Concreteness, Cue Phrase Presented, Part of Speech Changed, and Type of Change.

In addition to significant main effects for Concreteness and Type of Change, there were several significant interactions. The means for the significant interaction of Type of Change by Cue Phrase Presented are presented in Table $1(\mathrm{~F}=8.66, \mathrm{df}=2 / 56, \mathrm{p}<.001, \mathrm{MSe}=$ $.07)$. Two things are evident from this interaction. First, the fact that synonyms function as more effective distractors (see above) is seen here. Also, the relative effectiveness of using subject phrases, verb phrases, or object phrases as distractors is highlighted. Verb phrases where a synonym distractor is present pose the greatest problem to the subjects. A Scheffe test comparing the verb phrase to the noun phrases in the synonym condition was significant $(F=4.71$, df $=2 / 56, p<.02$, MSe $=.065)$. The means for the significant $(F=4.87$, df $=2 / 56, p<.02$, MSe $=.06$ ) interaction of Part of Speech Changed by Cue Phrase Presented, presented in Table 2, suggest that the source of this confusion with verb phrases lies with the verb itself. We can tentatively conclude that the strongest source of recognition errors lies with verb synonym distractors. This finding raises an interesting possibility. It is usually accepted that the reason for the effectiveness of synonyms as distractors is that long-term memory is organized semantically and that a synonym distractor in a recognition task confuses the subject, since he cannot differentiate it from his stored conception of the presented word. It may be that verbs are analyzed, comprehended, or stored differently from nouns (and other words) in sentence learning experiments.

If verbs are stored with more representation of the semantic structure present, then the results of Wearing (1970) are more readily interpretable. If verbs are primarily remembered by semantic structure, they should be less useful as retrieval cues (for surface structure) and also be more poorly recalled (verbatim). In

Table 1

Means for Interaction of Cue Phrase Presented by Type of Change

\begin{tabular}{lcc} 
& \multicolumn{2}{c}{ Type of Change } \\
& Synonym & Unrelated \\
\hline Subject & .74 & .79 \\
Verb & .56 & .88 \\
Object & .74 & .86 \\
\hline
\end{tabular}

Note-Entries are mean proportions correct (NEW phrases).

Table 2

Mean Proportions Correct (NEW Phrases) for Interaction of Part of Speech Changed by Cue Phrase Presented

\begin{tabular}{lcc} 
& \multicolumn{2}{c}{ Part of Speech Changed } \\
Modifier & Head \\
\hline Subject & .79 & .74 \\
Verb & .74 & .70 \\
Object & .74 & .86 \\
\hline
\end{tabular}


fact, both our intuitive feelings for the central importance of verbs in sentences and the importance stressed by theorists such as Chafe (1970) are supported by this kind of conception of the processing of verbs in comprehension and learning.

The recognition accuracy data may be rescored by treating each instance that a synonym distractor was falsely called OLD as a correct answer. If these data are averaged in with the correct recognition scores for truly OLD sentences, the probability of recognition of verb phrases is now .51 , while that of subject and object phrases is .45 and .48 , respectively. It is recognized that these are small differences and that Experiment I was not designed to test this effect. However, with respect to the values given above, the potency of the verb phrases as a cue was moved from last place to being at least equal to the noun phrases.

Another interesting finding is that, despite the strong difference between synonym and unrelated word changes, we failed to find an interaction between this factor and Concreteness, as reported by Begg and Paivio (1969). Since there was a significant four-way interaction involving Concreteness, Cue Phrase Presented, Part of Speech Changed, and Type of Change $(\mathrm{F}=5.18, \mathrm{df}=2 / 56, \mathrm{p}<.009, \mathrm{MSe}=.07)$ by collapsing across the appropriate factors, we can obtain results that may be compared to those of Begg and Paivio (Table 3). It appears from examination of Table 3 that subjects recognize not only meaning-altering changes but also meaning-preserving changes better for concrete material than for abstract material. This is just the reverse of the Begg and Paivio finding and will be discussed further below.

The results from this first experiment are far from being conclusive. With respect to the main question of what serves as the most easily recognized cue, the answer seems to be closely tied to how one scores the data. With a strict verbatim scoring procedure, nouns appear to be best, but if synonyms are accepted as correct, this advantage disappears. This finding is suggestive with respect to our second question of why one word may act as a better cue than others and how this is related to its form of storage. Perhaps verbs are processed to a semantically "deeper" level (in the generative semantic sense) and are therefore more easily confused with synonyms. As well, this offers a possible explanation as to why verbs are poorer cues for surface structure than nouns. Experiment II uses a forced-choice recognition paradigm to more rigorously test this question.

\section{EXPERIMENT II}

\section{Method}

Subjects. Twenty-four students from introductory psychology participated in the main experiment.

Design. This experiment used a forced-choice recognition memory paradigm where there were three possible answers presented at test time; these three answers included a phrase which actually had been presented in a preceding block of five sentences, a phrase where a verb (or noun) had been replaced by
Table 3

Proportion Correct Recognition of NEW Phrases as a Function of Concreteness, Type of Change (Synonym or Unrelated Word), and Part of Speech Changed (Verb or Noun)

\begin{tabular}{lcccc}
\hline & \multicolumn{2}{c}{$\begin{array}{c}\text { Nouns Changed } \\
\text { Synonym } \\
\text { Distractor }\end{array}$} & $\begin{array}{c}\text { Unrelated } \\
\text { Distractor }\end{array}$ & \multicolumn{2}{c}{$\begin{array}{c}\text { Verbs Changed } \\
\text { Synonym }\end{array}$} & $\begin{array}{c}\text { Unrelated } \\
\text { Distractor }\end{array}$ & Distractor \\
\hline Concrete & .83 & .89 & .66 & .91 \\
Abstract & .73 & .67 & .47 & .76 \\
\hline
\end{tabular}

a synonym, or a phrase where a verb (or noun) had been replaced by a different word, thus changing the meaning of the phrase. All manipulations used repeated-measures factors. These included Concreteness (concrete and abstract sentences), nature of the Cue Phrase Presented (subject phrase, verb phrase, or object phrase), and Type of Distractor (synonym or unrelated word).

Materials. The 24 critical sentences used were the same ones used in Experiment I. However, half of the altered sentences used in Experiment I had only synonym distractors available and the other half only unrelated words. Therefore, 12 new verb and noun synonyms and 12 unrelated verb and noun distractors were generated by the experimenters. These words were matched as closely as possible to the Thorndike-Lorge counts of the words for which they were substituted.

There exists the possibility that the effects noted in Experiment I are purely artifactual. In our choice of synonyms for nouns and verbs, we may have inadvertently picked verb synonyms which were closer in meaning to presented verbs than the noun synonym distractors were to the presented nouns. A brief rating experiment was performed to exclude this as an explanation. Eighteen volunteers from introductory psychology served as subjects. Each subject received a test booklet which contained all 24 sets of word-synonym sets and also all 24 sets of unrelated word sets chosen for Experiment II. Each subject rated the similarity in meaning of each presented word pair on a scale ranging from 1 (identical meaning) to 7 (completely different meaning). The possibility that the observed difference between synonym noun and verb substitution reported earlier is due to an artifact of material construction may be ruled out, because noun and verb synonyms were given almost the same mean rating ( 2.4 and 2.34 , respectively, n.s.).

Procedure. Testing took place in a group setting. Blocks of five sentences were presented visually for $20 \mathrm{sec}$ of study; after $10 \mathrm{sec}$ of an interpolated task involving crossing out letters, the subjects saw three phrases (same, synonym, unrelated) and recorded their judgment of which one they had actually seen in an answer booklet. Twenty-four trials were given.

\section{Results and Discussion}

Overall accuracy. There was only one significant effect when overall level of accuracy of picking the correct alternative was scored. Concrete phrases were more often correctly identified (.81) than abstract phrases (.58) $(\mathrm{F}=30.85, \mathrm{df}=1 / 20, \mathrm{p}<.001$, $\mathrm{MSe}=.05)$.

Error analysis. The frequency of false recognitions was analyzed in a 2 by 2 by 2 design, where the factors were Concreteness, Word Type (noun or verb), and Distractor Type (synonym or unrelated). The significant interaction of Word Type by Distractor Type $(F=5.01, \mathrm{df}=1 / 20, p=.03)$ clearly shows that synonym distractors are much more powerful for verbs than for nouns (see Table 4).

To return to the issue of the concreteness of the 
Table 4

Mean Proportion False Recognition of NEW Phrases as a Function of Word Type and Distractor Type

\begin{tabular}{lcc} 
& \multicolumn{2}{c}{ Distractor } \\
& Synonym & Unrelated Word \\
\hline Noun Phrase & .19 & .10 \\
Verb Phrase & .27 & .07 \\
\hline
\end{tabular}

materials and its effect on recognition memory: Our failure to replicate Begg and Paivio's (1969) finding in either Experiment I or II may be due to the differences in paradigm. Begg and Paivio presented their material verbally rather than visually and, on recognition trials, presented the whole sentence rather than just a cue phrase. Also, their method of changing a sentence's meaning was slightly different from ours: They interchanged the subject and object, while we substituted an unrelated word. If methodological variations are the cause of the lack of replication, then their finding is of only limited interest due to its lack of generality. The second possibility is that the distinction between concrete and abstract material is not qualitative, as is implied by Begg and Paivio's distinction in storage mechanism, but is rather due to some other factor(s), such as ease of comprehension. Thus, if both concrete and abstract material are handled by a single type of process, this could account for our finding that, although nouns and verbs differ in recognizability, the same relationship between the two holds for both concrete and abstract material.

Supporting evidence for a single mode of processing comes from a study of Tieman (1971). Tieman used comparative sentences of the form "The garage is newer than the theater" and manipulated the instructions given the subjects and the concreteness of the sentences. The subjects were instructed to try to remember the exact wording, the "gist" (i.e., meaning), or to form an image of the sentence referent and remember the image. A multiple-choice recognition test was used where two of the distractors were generated by interchanging subject and object nouns and replacing the comparative adjective with its opposite (e.g., older for newer). The amount of semantic confusion was the same over levels of concreteness, and the concreteness-abstractness of the adjectives did not interact with the instruction variable. This is in line with our findings from Experiments I and II.

Johnson, Bransford, Nyberg, and Cleary (1972) have criticized Begg and Paivio's study on several grounds: (1) Their concrete sentences were more easily comprehended than the abstract sentences, and (2) their test changes differentially affected the meaning of concrete as compared to abstract sentences. These two criticisms are also valid with respect to our stimulus material. The differential effect of our test change is indicated by the main effect for concreteness reported earlier; as well, on an intuitive level, our concrete sentences seem to be more easily comprehended than our abstract sentences.
Nonetheless, the result remains that there was no significant interaction between the concreteness of the materials and the type of change (meaning-preserving or meaning-altering) used in the distractors.

For both concrete and abstract sentences at a strictly verbatim (i.e., surface structure) level, it does appear that noun changes are more easily detected than verb changes. However, this effect may not be due to the noun playing the key role in sentence comprehension and storage; rather, it may be because the noun receives less semantic processing than verbs. This is indicated by the fact that verbs are more confused with words similar in meaning than nouns, as hinted in Experiment $I$ and clearly demonstrated in Experiment II. As well, it was found that this differential effect was not an artifact of the stimulus material used. Rather, it seems that in the processing of sentences, subjects actively analyze verbs to a deeper semantic level than nouns.

\section{EXPERIMENT III}

The finding that subjects appear to differentially process nouns and verbs leads one to question whether this is the result of a natural tendency on subjects' part or if it may reflect some general characteristics of the language. It is possible that within the language itself there exists some constraint which causes this observed difference. For example, the corpus of verbs available to describe any given action may have a higher overlap in meaning than the corresponding nouns. In order to obtain some tentative information on this question, a brief experiment was performed.

\section{Method}

Subjects. Thirty-two students from the same source as described earlier acted as subjects.

Procedure. This study was conducted in two parts; during the first stage, a number of photographs were taken of simple actor-action-object scenes (e.g., a hammer hitting a nail). Ten of the resulting slides that were judged least ambiguous were selected and presented to a group of 12 subjects. These subjects were instructed to generate two simple sentences of the form "The subject verbed the object," both of which adequately described the picture. They were also instructed that the two versions were to have no words in common, other than the definite article. These generated sentences were examined, and any instances where the same word appeared in both sentences or the grammatical frame was violated were discarded. From the pool of 91 remaining sentence pairs, the noun and verb pairs for each set were extracted. The resulting word pairs were then randomized and divided into two halves. Each half was presented to a group of 10 subjects with the instructions to rate the similarity in meaning of each word pair on a scale ranging from 1 (identical meaning) to 7 (completely different meaning). During the stage of sentence generation, the slides were presented in a standard-size classroom by means of a Kodak Carousel projector; subjects were given as much time as they needed to generate the two sentences and, in all instances, both sentences were generated in well under $2 \mathrm{~min}$, and usually in less than $1 \mathrm{~min}$. During the second stage of the study, the extracted word pairs were presented in booklets for other subjects to rate. There were nine word pairs to a page and, at assembly time, the page order for each booklet was scrambled. Subjects were allowed as much time as they needed for the rating task. 


\section{Results and Discussion}

Each subject's score for each picture-word pair combination was obtained, and these data were subjected to an analysis of variance. This analysis consisted of a 2 by 2 by 10 design, where the factors were Half (either the first or second half of the word pairs), Word Type (noun or verb), and Picture. The effect of major interest was that of Word Type, which was nonsignificant $(\mathrm{F}=4.11, \mathrm{df}=1 / 18, \mathrm{p}>.05, \mathrm{MSe}=.84)$. The similarity rating for nouns was 3.33 and that for verbs 3.52 . This is opposite of the possibility offered above and counter to any expectation formed on the bases of results from Experiments I and II. When noun synonym pairs and verb synonym pairs are presented in isolation, subjects tend to judge the nouns as being more similar in meaning than the verbs. However, as Experiments I and II demonstrated, when verb synonym distractors are presented in a sentence or phrase context, the reverse effect obtains.

Even though there was no difference between noun synonym pairs and verb synonym pairs on degree of rated similarity, there may still exist other important differences between the nouns and verbs generated by subjects. For example, the corpus of verbs available to describe some situations may have more commonality than nouns; there may not be as many available. Two analyses were performed to test this possibility. For each of the 10 slides, the number of different words (types) used to describe each of the three components of the situation (subject noun, verb, object noun) was computed. The sum across 10 slides was 62 for subject nouns, 68 for verbs, and 64 for object nouns. The second analysis examined the two most commonly used words for each of the three sentence positions. Summed across 10 slides, the two most commonly used words accounted for $62.5 \%$ of the words generated (tokens) for subject nouns, $62.3 \%$ for verbs, and $66.7 \%$ for object nouns.

It is clear from these two analyses that the verbs and nouns do not differ in the relative commonality of word choices.

\section{EXPERIMENT IV}

This study is a partial replication of Experiment II and was designed to insure greater orthographic and derivational similarity between noun and verb pairs. This study used noun and verb pairs which were derivationally related, that is, the stem of nouns and verbs was similar.

\section{Method}

Subjects. A total of 60 subjects participated in the study; 24 subjects served in the experiment proper, while 36 subjects helped generate filler sentences and rated semantic similarity of verb-verb and noun-noun pairs. All subjects were students in introductory psychology at Dartmouth College and served for course credit.

Design. The experiment used a recognition memory paradigm where seven sentences were presented in a block for a subject to study. After an interpolated activity (counting backward by threes), a sentence from the block of seven was presented to the subject, who had to signify if he had seen this sentence in the sample of seven sentences presented earlier. The experiment involved a factorial design with two repeatedmeasures factors; these factors were Part of Speech Changed (either the subject noun or the verb) and Type of Change (a change to a synonym or to an unrelated word). One-third of the test sentences presented were identical to a critical sentence presented in the block of seven sentences earlier, while twothirds of the test sentences involved a change in either the subject noun or the verb.

Materials. All sentences had the general form article, noun, verb, article, adjective, noun (e.g., The author cashed the large check). All sentences were relatively concrete, that is, the verbs and nouns in each sentence referred to a physical referent.

The experimenters generated a large number of word pairs whose stems could form the base of either verbs or nouns because of their derivational similarity; an example of such a pair would be sprinted-raced and sprinter-racer. Twenty-four subjects made counterbalanced semantic similarity judgments on the pairs of words; half the pairs were in the verb form and half in the noun form. From the initial pairs, 12 were selected which satisfied the double criteria of having similarity judgments above the neutral point (i.e., above 4 on a 7-point scale) and a good match on similarity judgments for the noun and verb forms. Table 5 presents the 12 pairs of words as well as the unrelated words used in each sentence, along with the mean synonymity judgments.

Procedure. Testing of subjects took place at a computer terminal connected to the Dartmouth time-sharing system. After the subject was seated at the terminal and had read the instructions, a block of seven sentences was presented. One of these seven was a target sentence; six were fillers. The fillers shared no words (other than articles) with each other or with the target sentence. This block was exposed for $30 \mathrm{sec}$, after which the subject was presented with a three-digit number and instructed to count backward by threes for 10 sec. At this time, the subject was presented with a test sentence which may or may not have been identical to one of the sentences in the preceding block of seven. Half the time the sentence presented was identical to one of the sentences presented earlier; half the time either the subject noun or the verb had been altered in some manner.

After the test sentence was presented, the subject was asked to decide whether he had seen this sentence in the preceding block of seven sentences (old/new judgment).

\section{Results and Discussion}

OLD cue phrases. The mean proportion correct for recognizing OLD sentences was .80 ; thus, subjects were reasonably accurate in recognizing truly old or unchanged sentences.

NEW cue phrases. The proportions wrong for judging NEW sentences were analyzed in a two-way analysis of variance where the two factors were Part of Speech Changed and Type of Change.

In addition to two highly significant main effects, there was a significant interaction between the two factors $(F=6.24, \mathrm{df}=1 / 23, \mathrm{p}=.02, \mathrm{MSe}=.07)$. Table 6 presents the mean proportions wrong for recognition of new sentences; as can be seen from the table, verb synonyms function as much more effective distractors than noun synonyms. This effect has been seen previously in Experiments I and II, but here, where there is more adequate control of orthographic and derivational similarity between nouns and verbs, the effect 
Table 5

Stimulus Sentences Used in Experiment $V$

1 (a). The janitor (washed/cleaned/broke) the dirty window.

1 (b). The (washing/cleaning/pumpkins) filled the whole basket.

2 (a). The woman (acted/performed/memorized) the difficult part.

2 (b). The (acting/performing/skating) required a night rehearsal.

3 (a). The child (punted/kicked/caught) the old football.

3(b). The (punt/kick/lineman) saved the football game.

4 (a). The father (labored/worked/smoked) the whole period.

4 (b). The (laborer/worker/father) dropped the heavy stone.

5 (a). The athlete (sprinted/raced/dreaded) the last mile.

5 (b). The (sprinter/racer/soldier) completed the last mile.

6 (a). The corporation (transferred/moved/upset) the young executive.

6(b). The (transfer/move/secretary) upset the young executive.

7 (a). The student (aided/helped/heard) the injured man.

7 (b). The (aid/help/studying) caused the final success.

8 (a). The millionaire (walked/strolled/reached) the long street.

8 (b). The (walk/stroll/spices) heightened the depressed appetite.

9 (a). The reporter (counseled/advised/interviewed) the innocent man

9 (b). The (counselor/adviser/reporter) interviewed the innocent man.

10 (a). The professor (taught/instructed/hated) the young student.

10(b). The (teacher/instructor/mechanic) sought the best results.

11 (a). The teenager (authored/wrote/read) the lurid novel.

11 (b). The (author/writer/teller) cashed the large check.

12 (a). The substitute (refereed/umpired/played) the close game.

12 (b). The (referee/umpire/announcer) called the close play.

Note-Words in parentheses are the synonym pair and different distractor. The number following the sentence is the mean similarity rating for the synonym pair ( $7=$ identical).

Table 6

Mean Proportion Wrong Recognition of

Synonym and Unrelated Distractors

\begin{tabular}{lcc} 
& \multicolumn{2}{c}{ Distractor Type } \\
& Synonym & Unrelated \\
\hline Verbs & .396 & .021 \\
Nouns & .125 & .021 \\
\hline
\end{tabular}

is even more striking. For different word distractors, nouns and verbs are equal in judged oldness. However, noun synonyms are incorrectly judged old only about $13 \%$ of the time, while those test sentences containing verb synonyms are incorrectly judged old almost $40 \%$ of the time.

\section{EXPERIMENT V}

The tentative explanation which was offered for the results of Experiments I, II, and IV was that verbs, when encoded, retain more semantic information than nouns. This structural difference in memory encoding between verbs and nouns is postulated to cause the high degree of false recognition of verb synonyms in a later recognition test. However, there is a highly plausible alternative explanation for the obtained data. It may be that nouns and verbs are processed to equivalent semantic levels, and are encoded to a similar depth; however, for nouns more surface structure or orthographic information is retained. This alternative explanation would suggest when a synonym distractor is presented at test time, the memory representation for nouns would have two components: semantic information and orthographic or feature information. Verbs, on the other hand, are remembered only in respect to semantic components. If this is the case, then the obtained data which show a high degree of false recognition for verb synonyms is due, not to the fact that verbs are processed to a deeper semantic level, but rather verbs and nouns are processed to equivalent semantic levels, but a secondary source of information is retained for nouns. This means that, when a noun synonym is presented as a distractor, a subject is less likely to "recognize" this noun synonym as being old because, in fact, he has two sources of information to tell him that this noun synonym distractor is, in fact, new. Thus, the obtained differences between verb and noun synonym distractors may be due to the fact that subjects are simply more accurate for noun synonym distractors because they have more information available to them.

This alternative explanation offers one clear prediction in terms of the amount of semantic information available to subjects for verbs and nouns. If this alternative explanation is true, then verbs and nouns are encoded in a similar semantic fashion, and a rating task performed immediately after the presentation of sentences should show verb and noun synonymity ratings to be equal. Experiment $\mathrm{V}$ was designed to test whether subjects perceive sentences with verb and noun synonym distractors as being equally synonymous with sentences presented in the acquisition phase of the experiment.

\section{Method}

With two exceptions, the materials and general procedure in this study were identical to those of Experiment IV. During the test phase of the experiment, subjects were asked to rate the degree of semantic similarity of the presented test sentence to the corresponding sentence which had been presented in the earlier block of seven sentences instead of making an old/new judgment as in Experiment IV. As well, there was no interpolated activity between the block of seven sentences and the test phase. Twenty-four subjects from introductory psychology participated in this study.

\section{Results and Discussion}

The main dependent variable of interest is judgment of similarity of sentences on a 1 to 7 scale, where 7 
means identical semantic content, or total synonymity. For the 12 truly old sentences which each subject saw, the mean similarity rating was 6.05 . On the other hand, for the six instances where unrelated words were substituted, the mean similarity rating was 3.0 for verbs and 2.88 for nouns (n.s.). When test sentences involving synonyms were presented, the mean similarity rating for verbs was 5.52 and for nouns $4.68(t=2.62, d f=23$, $\mathrm{p}<.008$, sed $=.32$ ). These data are in sharp contrast to the mean similarity ratings for these pairs obtained in isolation (outside of sentences) in Experiment IV; those mean synonymity ratings were 5.40 for verb synonym pairs and 5.35 for noun synonym pairs.

The results of this study demonstrate clearly that, as far as subjective impressions of semantic similarity are concerned, test sentences containing verb synonyms are more closely related, semantically, than test sentences involving noun synonyms. This is in direct contradiction to the prediction stated above, which maintained that noun synonyms and verb synonyms should contain equivalent amounts of semantic information.

\section{GENERAL DISCUSSION}

As was pointed out in the introduction, several theoretical trends point to the verb's importance in the sentence. This view is corroborated by rating and sorting experiments (e.g., Healy \& Miller, 1970) and by processing tasks that directly manipulate verb characteristics (e.g., Rohrman, 1970).

A problem arises in reconciling these facts with the general inferiority of verbs in memorial tasks (e.g., James, 1972; Wearing, 1970, 1973); Wearing (1974) has called this problem "Fillenbaum's paradox."

It was proposed that three classes of models exist as explanations of Fillenbaum's paradox. The "verb decomposition model" is clearly wrong. Experiments I and II showed that verbs were stored with more semantic content than nouns, as indicated by the higher false positive response rate to synonym distractors. Since this effect was noted with isolated verb phrases, rather than for intact sentences, the nouns could not be acting as repositories of meaning for "decomposed" verbs. It might be worthwhile to note here that quasi-F analyses (Clark, 1973) were performed where appropriate throughout the analyses in the present experiments and failed to alter any of the conclusions reached through conventional analyses. Hence, the present results may be generalized over both sentences and subjects.

The "task demand model" also appears to be wrong. The results of Experiments I, II, IV, and, especially, $\mathrm{V}$ indicate that the verbs in presented sentences do not receive less semantic analysis at presentation time. If anything, they seem to receive more semantic analysis, as indicated by the high synonymity ratings given in Experiment V.
This leaves us with some version of the "semantic encoding model." It appears that Wearing's (1974) speculation was partially correct; verbs do change storage form in the course of the memory task, and they do lose some surface structure representation. For purpose of analysis, we can imagine two main components in the memory storage of individual wordssemantic information and form information (orthographic or phonological features). It may be that verbs and nouns retain equal amounts of semantic information or are processed to equal semantic levels, but nouns retain more form information. This would explain the high false positive rate for synonyms but is directly contradicted by the rating data of Experiment $V$.

Another possibility is that verbs are processed to a deeper semantic level but nouns and verbs retain equivalent amounts of form information. This pattern would nicely explain the present pattern of results but is totally incompatible with the variety of earlier studies on recognition and recall of verbs and nouns.

We are forced to conclude that a complex processing interaction occurs at comprehension time. Verbs are processed to a deeper semantic level and nouns have the form information encoded more strongly. In Craik and Lockhart's (1972) terms, verbs undergo a more extensive Type II analysis, while nouns receive an extended Type I analysis. These two independent foci of analysis will yield differential storage strengths for the two word classes. It is important to note that both of these differential analyses must be hypothesized in order to explain both the present pattern of results and those of earlier memorial studies.

While an application of "levels of processing" has been demonstrated clearly by Graesser and Mandler (1975) for surface and idea structure of entire sentences, only Gomulicki (1956) appears to have offered an intrasentence example. In fact, the essential elements of the semantic encoding model were proposed 20 years ago by Gomulicki. He noted: “. . . the abstractive process seems to develop concurrently with the process of understanding a passage while hearing it [and] the selectivity of mnemic abstraction implies an unwitting ranking of parts according to importance" (p. 90). He went on to suggest that "... attention fluctuates during the hearing of a passage, certain parts drawing 'maximum attention' at the expense of others ..." (p. 90).

The semantic encoding model nicely explains the present pattern of results and most of the data on verb and noun recall in the literature. However, three studies where the results seem incompatible with the model must be examined in more detail.

The most troublesome data to explain is that of Thios (1975). He found that subject nouns were better cues for sentence recall than either verbs or object nouns which did not differ from each other in effectiveness. This argues that there was no differential storage for verbs and object nouns. However, several facets of the 
analysis prevent easy comparisons. First, the dependent measure was proportion of complete sentence recalls. Thus, subject nouns were acting as cues for verbs and object nouns, verbs were acting as cues for two nouns, and object nouns were acting as cues for a verb and the subject noun. The form of storage of the verb is difficult to determine with this complex set of interrelations. Second, Thios found that, when verb synonyms were allowed as correct answers, "general" sentences were more affected by this more liberal scoring criterion than were sentences with "specific" verbs. However, this is probably artifactual since general verbs, almost by definition, have more synonyms available.

Wearing (1971) reported, in a divided attention task with immediate recall, that recall of verbs was superior to that of nouns. First, only one statistical test of verbnoun recall (out of three possible) was reported. As well, the failure of recall of nouns due to high levels of within-sentence interference is a real possibility; this possibility is further complicated by a strong serial position effect noted by Wearing. Lastly, it is difficult, if not impossible, to judge the nature of the memory storage for the elements of these sentences because of the possibility of a variety of recall cues (phonological, semantic, etc.) being available in the immediate test paradigm.

The last study to be examined is that of Wearing (1974), who found no difference in verb-noun recall in low-potency sentences but a significant decrement for high-potency verbs. On the surface, it is difficult to see how the semantic encoding model would predict this differential effect. However, if high-potency verbs are more important to a sentence, they will be analyzed to a deeper semantic level, and will come out poorly on a verbatim test of recall; this is predictable from the semantic encoding model.

A second model may be proposed which is conceptually similar to the semantic encoding model. It may be that verbs and nouns are processed to equivalent semantic and form levels but that differential decay of the word attributes occurs for some other reasons. These may be related to interfering effects of other stimulus materials, the mere passage of time, etc. A series of experiments is currently underway in an attempt to separate these two similar explanations.

The theatrical metaphor of Healy and Miller (1970) appears still to be well chosen and is supported by the present series of experiments. The plot is subjected to a deeper "semantic" analysis by both audiences and theater critics; classic plays become immortal because of the timelessness of their message rather than the crude mechanics of their entrances, exits, and scene lengths, all of which seem soon forgotten. Actors, on the other hand, serve as interpreters of a plot; their effectiveness and fame rests on more immediate stylistic devices. Although they too have a "semantic level," it is either not comprehended or else quickly forgotten.

\section{REFERENCE NOTE}

1. Townsend, D., \& Saltz, E. Recall of sentences: Comparison of phrase structure vs. core meaning theories. Paper read to the Midwestern Psychological Association, 1972.

\section{REFERENCES}

Anderson, R. C., \& Hidde, J. L. Imagery and sentence learning. Journal of Educational Psychology, 1971, 62, 526-530.

Bacharach, V. R., Kellas, G., \& McFarland, C. E. Structural properties of transitive and intransitive verbs. Journal of Verbal Learning and Verbal Behavior, 1972, 11, 486.490.

BegG, I., \& Paivio, A. U. Imagery and sentence meaning. Journal of Verbal Learning and Verbal Behavior, 1969, 8, 821.827.

Blumenthal, A. L. Prompted recall of sentences. Joumal of Verbal Learning and Verbal Behavior, 1967, 6, 203-206.

BOWER, G. H. Imagery as a relational organizer in associative learning. Joumal of Verbal Leaming and Verbal Behavior. $1970,9,529-533$.

CHAFE. W. Meaning and the structure of language. Chicago: University of Chicago Press, 1970.

Clark, H. H. The language-as-fixed-effect fallacy: A critique of language statistics in psychological research. Journal of Verbal Learning and Verbal Behavior, 1973, 12, 335-359.

CRAIK, F. I. M., \& LockharT, R. S. Levels of processing: A framework for memory research. Journal of Verbal Learming and Verbal Behavior, 1972, 11, 671-684.

Fillenbaum, S. On the use of memorial techniques to assess syntactic structures. Psychological Bulletin. 1970, 73, 231-237.

Fodor, J. A., Garrett. M., \& Bever, T. G. Some syntactic determinants of sentential complexity: II. Verb structure. Perception \& Psychophysics, 1968, 3, 453-461.

Gladney, T. A., \& Krulee, G. K. The influence of syntactic errors on sentence recognition. Journal of Verbal Leaming and Verbal Behavior, 1967, 6, 694-698.

Gollub. H. F. Impression formation and word combination in sentences. Journal of Personality and Social Psychology, $1968,10,341-353$.

Gomulick1, B. R. Recall as an abstractive process. Acta Psychologica, 1956, 12. 77.94.

Graesser, A., \& MANOLER, G. Recognition memory for the meaning and surface structure of sentences. Journal of Experimental Psychology: Human Learning and Memory, $1975,104,238-248$.

Healy, A. F., \& Miller, G. A. The verb as the main determinant of sentence meaning. Psychonomic Science, 1970, 20, 372.

HeAly, A. F., \& Miller, G. A. The relative contribution of nouns and verbs to sentence acceptability and comprehensibility. Psychonomic Science, 1971, 24, 94-96.

HEISE, D. R. Affectual dynamics in simple sentences. Journal of Personality and Social Psychology, 1969, 11, 204-213.

HEISE, D. R. Potency dynamics in simple sentences. Journal of Personality and Social Psychology, 1970, 16, 48-54.

Horowitz, L. M.. \& Prrtulak, L. S. Redintegrative memory. Psychological Review, 1969, 76, 519-531.

JAMES, C. T. Theme and imagery in the recall of active and passive sentences. Journal of Verbal Learning and Verbal Behavior, 1972, 11, 205-211.

JARVElla, R. J. Syntactic processing of connected speech. Journal of Verbal Learning and Verbal Behavior, 1971, 10, 409.416.

Johnson, M. K., Bransford, J. D., Nyberg, S. F., \& Cleary, J. J. Comprehension factors in interpreting memory for abstract and concrete sentences. Journal of Verbal Learning and Verbal Behavior, 1972, 11, 451-454.

Kintsch, W. Notes on the structure of semantic memory. In 
E. Tulving, \& W. Donaldson (Eds.), Organization of memory. New York: Academic Press. 1972.

KinTsCH, $W$. The representation of meaning in memory. New York: Wiley, 1974.

MARTIN, E., \& RoBeRTS, K. H. Grammatical factors in sentence retention. Journal of Verbal Learning and Verbal Behavior, 1966, 5, 211-218

Martin, E., Roberts, K. H., \& Collins, A. M. Short-term memory for sentences. Journal of Verbal Leaming and Verbal Behavior. 1968, 7, 560-566.

MARTIN, E., \& WALTER, D. A. Subject uncertainty and wordclass effects in short-term memory for sentences. Journal of Experimental Psychology, 1969, 80, 47-51.

Polzella, D. J., \& Rohrman, N. L. Psychological aspects of transitive verbs. Joumal of Verbal Learning and Verbal Behavior, 1970, 9.537.540.

REID, L. S. Toward a grammar of the image. Psychological Bulletin, 1974, 81, 319-334.

RotrRman, N. L. More on the recall of nominalizations. Journal of Verbal Learning and Verbal Behavior, 1970, 9, 534-536.

Rumelhart, D. E., Lindsay, P. H., \& Norman, D. A. A process model for long-term memory. In E. Tulving, \& W. Donaldson (Eds.), Organization of memory. New York: Academic Press, 1972.

SACHS, J. S. Recognition memory for syntactic, and semantic aspects of connected discourse. Perception \& Psychophysics, $1967,2,437-442$.

Thios. S. J. Memory for general and specific sentences. Memory \& Cognition, 1975, 3, 75-77.
TIEMAN, D. G. Recognition memory for comparative sentences. Unpublished doctoral dissertation, Stanford University, 1971. In I. R. Anderson \& G. H. Bower (Eds.), Human associative memory. New York: Wiley, 1973.

WEARING, A. J. The storage of complex sentences. Joumal of Verbal Learning and Verbal Behavior, 1970, 9, 21-29.

Wearing, A. J. Word class and serial position in the immediate recall of sentences. Psychonomic Science, 1971, 25, 338-340.

Wearing, A. J. Remembering complex sentences. Quarterly Journal of Experimental Psychology, 1972, 24, 77-86.

WEARING, A. J. The recall of sentences of varying length. Australian Journal of Psychology, 1973, 25, 155-161.

WEARING, A. J. Task differences and word-class effects in sentence processing. Australian Journal of Psychology, 1974, 26. 131-138.

Wearing, A. J., \& Crowder, R. G. Dividing attention to study sentence acquisition. Journal of Verbal Leaming and Verbal Behavior, 1971, 10, 254-261.

WEISBERG, R. Sentence processing assessed through intrasentence word associations. Joumal of Experimental Psycho$\log y, 1969,82,332-338$.

YNGVE, V. H. A model and a hypothesis for language structure. Proceedings of the American Philosophical Society, 1960. 104, 444-466.

(Received for publication August 25, 1975; revision accepted November 13,1975 .) 